\title{
Group Classification of a generalization of the Heath Equation
}

\author{
Y. Bozhkov ${ }^{\mathrm{a}}$, S. Dimas ${ }^{\mathrm{a}, *}$ \\ ${ }^{a}$ Instituto de Matemática, Estatística e Computação Científica - IMECC \\ Universidade Estadual de Campinas - UNICAMP, Rua Sérgio Buarque de Holanda, 651 \\ 13083-859 - Campinas - SP, Brasil
}

\begin{abstract}
The complete group classification of a generalization of the Heath model is carried out by connecting it to the heat equation with nonlinear source. Examples of invariant solutions are given under the terminal and the barrier option condition.
\end{abstract}

Keywords: Heath equation, Lie point symmetry, group classification, barrier option, computer assisted research 2010 MSC: 35A20, 70G65, 70M60, 97M30, 68W30

\section{Introduction}

The area of Financial Mathematics was founded by the pioneering works of Samuelson, Modigliani, Merton, Black and Scholes with the formal introduction of the concepts of stochastic calculus into Econometrics. Through the modeling process of incorporating the randomness into a deterministic model a set of evolutionary PDEs is obtained. Amongst them are:

- The Black-Scholes-Merton Equation [1],

$$
u_{t}+\frac{1}{2} \sigma^{2} x^{2} u_{x x}+r x u_{x}-r u=0,
$$

\footnotetext{
*Corresponding author

Email addresses: bozhkov@ime.unicamp.br (Y. Bozhkov), spawn@math.upatras.gr (S. Dimas)
} 
- The Longstaff Equation [2],

$$
u_{t}-\left(\frac{1}{4} \sigma^{2}-\kappa \sqrt{x}-2 \lambda x\right) u_{x}-\frac{1}{2} \sigma^{2} x u_{x x}+x u=0,
$$

- The Vasicek Equation [3],

$$
u_{t}+\frac{1}{2} \sigma^{2} u_{x x}-(\kappa-\lambda x) u_{x}-x u=0,
$$

- The Cox-Ingersoll-Ross Equation [4],

$$
u_{t}+\frac{1}{2} \sigma^{2} x u_{x x}-(\kappa-\lambda x) u_{x}-x u=0,
$$

- A Hamilton-Jacobi-Bellman type Equation [5],

$$
u_{t}+\alpha u_{x}+\frac{1}{2} b^{2} u_{x x}-\frac{1}{2} u_{x}^{2}+\nu(x)=0,
$$

where $u=u(x, t)$. Eqs. (1)-(4) have similar structure, in fact, they are connected with the Heat Equation,

$$
u_{t}=u_{x x}
$$

via an invertible transformation as first shown for Eq. (1) in [6] and later for the rest of them in [7]. Actually, Eqs. (2)-(4) are encompassed in a general bond-pricing equation presented in [8]. Furthermore, it is shown that Eqs. (1)-(4) can be contained in a nonlinear generalization of this bondpricing equation [9].

The last of the above equations, (5), was first presented by Heath et al in [5] as an equation for mean variance hedging. Even though at first glance it looks to be different in structure than the rest, it can be linearized, and actually, for some specific cases of $\nu(x)$ can be linked with (6) as well [10, 7].

For all the aforementioned connections of the Eqs. (1)-(4) with the heat equation the use of symmetries was prominent. The symmetry analysis of differential equations is a method first developed in the $19^{\text {th }}$ century by Sophus Lie. One of the main benefits of this method is that by following a completely algorithmic procedure one is able to determine the symmetries of a differential equation or systems of differential equations. Grosso modo, the 
symmetries of a differential equation transform solutions of the equation to other solutions. The Lie point symmetries comprise a structural property of the equation - in essence they are the DNA of an equation. The knowledge of the symmetries of an equation enables one to utilize them for a variety of purposes, from obtaining analytical solutions and reducing its order to finding of integrating factors and conservation laws. In fact, many, if not all, of the different empirical methods for solving ordinary differential equations (ODEs) we have learned from standard courses at the undergraduate level emerge from a symmetry. For instance, having at our disposal a Lie point symmetry of a first order ODE, we can immediately get explicitly an integrating factor. Furthermore, even the knowledge of a trivial solution of the equation can be used for creating nontrivial solutions by using the equation's symmetries. And all these are due to the rich underlining algebraic structure of Lie groups and Lie algebras with which we give flesh to the symmetries of a differential equation.

Another important characteristic of the symmetry method is that in some situations the symmetries of an equation may indicate that it can be transformed to a linear equation, to be linearized. In addition, its symmetries provide the means to construct the needed transformation. A strong indication for that is the existence of an infinite dimensional Lie algebra, [11].

Moreover, a valuable tool when considering classes of equations containing arbitrary constants and functions is the use of the equivalence or admissible transformations, $[12,13,14]$. Equivalence transformations of a class of differential equations are point transformations that keep this class invariant, in other words they map an equation from this class to another member of the same class. In the recent years equivalence transformations have found much application either as a stand alone analytic tool for the group classification of differential equations, $[15,16,9]$, or at the core of the enhanced group analysis, [17, 18, 19, 20].

In the present work the following generalization of Eq. (5),

$$
u_{t}=a u_{x}+\frac{1}{2} u_{x}^{2}-\frac{1}{2} b^{2} u_{x x}+f(x, u), b, f_{u} \neq 0
$$

is studied under the prism of symmetry analysis. It is evident that in general Eq. (7) will not be linearizable as Eq. (5).

Furthermore, two distinct conditions will be considered along with (7). The terminal condition

$$
u(x, T)=1
$$


where $T$ is the terminal time, and the barrier option condition

$$
\begin{aligned}
& u(H(t), t)=R(t), \\
& u(x, T)=\max (x-K, 0),
\end{aligned}
$$

where the barrier option $u(x, t)$ satisfies Eq. (7) for $x>H(t), t<T{ }^{1}$ The constant $T$ again is the terminal time where the barrier option is exercised and $K$ is the strike price.

The first condition (9a) describes the evolution of standard or "vanilla" products; the price of a zero-coupon bond (or of a financial option), $u(x, t)$, which expires when $t=T[21,1,22,23]$.

The second condition (9b) takes into account the possibility of an unacceptable change in the price of the underlying option. It is considered an exotic type of option possessing features that makes it more complex than the "vanilla" option, [24, 25, 26]: The underlying idea is that now a barrier $H(t)$ exists and when the asset price $x$ crosses it, the barrier option $u(x, t)$ becomes extinguished or comes into existence. Those two types are also known as down-and-out and down-and-in, respectively. Often a rebate, $R(t)$, is paid if the option is extinguished. In what follows we shall consider the down-and-out type. A common assumption for the barrier function $H$ is to have the exponential form

$$
H(\tau)=\beta K e^{-\alpha \tau},
$$

where $\alpha \geq 0,0 \leq \beta \leq 1$ and $\tau=t-T$ [25, p. 187].

The main purpose of this paper is to carry out the complete group classification of Eq. (7) and construct analytical solutions satisfying one of the two conditions. Recall that to perform a complete group classification of a differential equation (or a system of differential equations) involving arbitrary functions or/and parameters, means to find the Lie point symmetry group $\mathcal{G}$ for the most general case, and then to find specific forms of the differential equation for which $\mathcal{G}$ can be enlarged, [27, p. 178]. Quite often, there is good physical or geometrical motivation to study such cases. Indeed, the larger the group the bigger the number of symmetries that will "survive" after imposing the conditions and hence the greater the chance of constructing an

\footnotetext{
${ }^{1}$ It is evident that the terminal condition (8) is included as a special situation when the asset price exceeds a value defined by the barrier function $H$ on time $T$.
} 
analytical solution corresponding to a more realistic financial model - in our case - following more closely the complex evolution of the financial option or bond. This is particularly true for the case of the barrier option as recent results have shown $[26,16]$.

As previously mentioned the key analytical tool used in this work is the symmetry analysis of Eq. (7). One of the advantages of this approach, as already emphasized, is that it provides a well defined algorithmic procedure which essentially enables one to find the involved linearizing transformations, conservation laws, invariant solutions, etc. On the other hand, the calculations involved are usually very difficult and extensive even for the simplest equations. Thus, it may become very tedious and error prone. For this reason the real progress in this area occurred in the last few decades with the advances in computer technology and the development of computer algebra systems like Mathematica, Maple, Reduce, etc. Based on these systems, a handful of symbolic packages for determining the symmetries of differential equations exists, [28, 29, 30,31]. Such a symbolic package, based on Mathematica, [32], has been developed by S. Dimas. The package, named SYM, $[33,34,35]$, was developed from the ground up using the symbolic manipulation power of Mathematica and the artificial intelligence capabilities which it offers. It was extensively used for all the results in the present paper, both for the interactive manipulation of the found symmetries and for the classification of the equations employing the symbolic tools provided by it.

This paper is organized as follows. In section 2 the basic concepts of the Lie point symmetry approach to differential equations used in the paper are presented. In section 3 we show how Eq. (7) can be connected with the heat equation with nonlinear source and hence reduce the problem of the complete group classification to it. In section 4 we give various examples of how the classification can be used in order to obtain similarity solutions under the specific boundary problems studied, the "vanilla" option and the barrier option. Finally, in section 5 the obtained results in this work are discussed.

\section{Preliminaries}

In this section we expose some notions of the modern group analysis that will be encountered in the main sections of the article suitably adapted to the article's needs. For a full treatise of the subject we direct the interested reader to the classical texts $[27,11,36,12,37,38]$. 
A Lie point symmetry ${ }^{2}$ of (7) is a differential operator, named infinitesimal generator,

$$
\mathfrak{X}=\xi^{1}(x, t, u) \frac{\partial}{\partial x}+\xi^{2}(x, t, u) \frac{\partial}{\partial t}+\eta(x, t, u) \frac{\partial}{\partial u},
$$

that satisfy the condition

$$
\left.\mathfrak{X}^{(2)}\left[a u_{x}+\frac{1}{2} u_{x}^{2}-\frac{1}{2} b^{2} u_{x x}+f(x, u)-u_{t}\right]\right|_{(7)} \equiv 0
$$

where $\mathfrak{X}^{(2)}$ is the suitable prolongation of the differential operator up to order two. The set of all the symmetries admitted by a differential equation constitutes a Lie algebra.

Having the symmetries there is a wealth of things that can be done with. In the present paper, we use them to obtain invariant or similarity solutions of the Eq. (7). By invariant solutions we mean solutions of (7) that are invariant under one of the found symmetries $\mathfrak{X}$, that is,

$$
\left.\mathfrak{X}[u-\varphi(x, t)]\right|_{u=\varphi(x, t)} \equiv 0 .
$$

The Eq. (12) is a linear PDE called invariant surface condition and by solving it we obtain a way to reduce the order of Eq. (7). Similarly, when we look for a similarity solution of Eq. (7) along with a initial/boundary condition we have to choose the subalgebra leaving also invariant that condition and its boundary:

$$
\left.\mathfrak{X}(t-T)\right|_{t=T} \equiv 0
$$

and

$$
\left.\mathfrak{X}(u-1)\right|_{t=T, u=1} \equiv 0
$$

for the boundary condition (8). And

$$
\begin{gathered}
\left.\mathfrak{X}(x-H(t))\right|_{x=H(t)} \equiv 0, \\
\left.\mathfrak{X}(u-R(t))\right|_{x=H(t), u=R(t)} \equiv 0
\end{gathered}
$$

for the boundary condition (9).

Another useful notion is that of additional equivalence transformation. An additional equivalence transformation is a point transformation that connects

\footnotetext{
${ }^{2}$ Henceforth referred simply as symmetry.
} 
inequivalent - from the point of view of equivalence transformations subclasses of a broader class of differential equations, which contains the class of differential equations under consideration, that possess the same Lie algebra of symmetries. The knowledge of such transformations greatly facilitates the classification.

\section{Group classification}

In this section we proceed with the group classification of Eq. (7). First, we observe that the point transformation

$$
\tau=-\frac{b^{2}}{2} t, \phi=\exp \left(-\frac{1}{b^{2}}(a x+u)\right)
$$

turns the class (7) into the heat equation with nonlinear source,

$$
\phi_{\tau}=\phi_{x x}+\hat{f}(x, \phi), \hat{f}_{\phi \phi} \neq 0
$$

where $\hat{f}=\frac{1}{b^{4}} \exp \left(-\frac{1}{b^{2}}(a x+u)\right)\left(2 f-a^{2}\right)$. Hence the problem of the complete group classification of the class $(7)$ is reduced to the group classification of the heat equation with nonlinear source, (18).

Remark 3.1. One can arrive to (17) either by looking for the best representative for (7) by utilizing its equivalence algebra to zero out as many arbitrary elements as possible, or by using the Hopf-Cole transformation in combination with a linear transformation since (7) is very similar to the Burgers equation in potential form and the well established fact in the literature that Eq. (5) is connected with the Heat equation.

Remark 3.2. It is evident that if the function $f$ has the form $f(x, u)=$ $\frac{1}{2}\left(\exp \left(\frac{1}{b^{2}}(a x+u)\right) g(x)+a^{2}\right)$, where $g$ an arbitrary real function of $x$, then Eq. (7) is linearizable.

To classify the class of equations (18) we analyzed the classification equation,

$$
\begin{aligned}
& \hat{f}_{\phi}\left(\mathcal{F}_{1}(x, t)+\frac{1}{8} \phi\left(8 \mathcal{F}_{4}(t)+x\left(x \mathcal{F}_{2}^{\prime \prime}(t)-4 \mathcal{F}_{3}^{\prime}(t)\right)\right)\right)+\mathcal{F}_{1, x x}(x, t)- \\
& \frac{1}{8} \hat{f}\left(8 \mathcal{F}_{4}(t)+x\left(x \mathcal{F}_{2}^{\prime \prime}(t)-4 \mathcal{F}_{3}^{\prime}(t)\right)\right)+\hat{f}_{x}\left(\mathcal{F}_{3}(t)-\frac{x \mathcal{F}_{2}^{\prime}(t)}{2}\right)+\frac{1}{4} \phi \mathcal{F}_{2}^{\prime \prime}(t)- \\
& \quad-\hat{f} \mathcal{F}_{2}^{\prime}(t)-\mathcal{F}_{1, t}(x, t)+\frac{1}{8} \phi\left(x\left(4 \mathcal{F}_{3}^{\prime \prime}(t)-x \mathcal{F}_{2}^{\prime \prime \prime}(t)\right)-8 \mathcal{F}_{4}^{\prime}(t)\right)=0
\end{aligned}
$$


derived from the symmetry condition of (18).

This classification is included in the work of Zhdanov et al. [39]. In their work, the group classification of the heat conductivity equation with a nonlinear source,

$$
u_{t}=u_{x x}+F\left(t, x, u, u_{x}\right),
$$

was performed taking advantage of the fact that the abstract Lie algebras of dimensions up to five are already classified.

Because of that fact we refrain any further details of the calculations involved and proceed by giving the classification modulo the equivalence group $G^{\sim}$,

$$
\tilde{x}=\delta_{4} x+\delta_{3}, \tilde{\tau}=\delta_{4}^{2} \tau+\delta_{0}, \tilde{\phi}=\delta_{1} \phi+\mathcal{F}(x), \delta_{4} \neq 0,
$$

of the class (18).

In Table 1 the Lie algebra for each form of the function $\hat{f}$ is given according to the following notations:

$$
\begin{aligned}
& A_{1}=\operatorname{span}\left(\partial_{\tau}\right) \text {, } \\
& A_{2,2}^{1}=\operatorname{span}\left(\partial_{\tau}, e^{\tau} x \partial_{x}+2 e^{\tau} \partial_{\tau}-e^{\tau}\left(\frac{1}{4} x^{2}+A\right) \phi \partial_{\phi}\right) \text {, } \\
& A_{2,2}^{2}=\operatorname{span}\left(\partial_{\tau}, x \partial_{x}+2 \tau \partial_{\tau}-B \phi \partial_{\phi}\right) \text {, } \\
& A_{2,2}^{3}=\operatorname{span}\left(\partial_{\tau}, 2 e^{2 A \tau} \partial_{x}-e^{2 A \tau}(2 A x+B) \phi \partial_{\phi}\right) \text {, } \\
& A_{2,2}^{4}=\operatorname{span}\left(\partial_{\tau}, e^{-A \tau} \phi \partial_{\phi}\right) \text {, } \\
& A_{3,5}^{1}=\operatorname{span}\left(\partial_{\tau}, e^{2 A \tau} \partial_{x}-e^{2 A t}\left(A(x+\Delta) \phi+\frac{2 E x^{\frac{2}{B+1}-1} e^{-\frac{1}{2} A(x+\Delta)^{2}}}{B+1}\right) \partial_{\phi},\right. \\
& 2 e^{4 A \tau} A(x+\Delta) \partial_{x}+e^{4 A \tau} \partial_{\tau}-\frac{2 A e^{4 A t}}{B+1}\left(\left(A(B+1)(x+\Delta)^{2}-2\right) \phi+\right. \\
& \left.\left.2 \Delta E x^{\frac{2}{B+1}-1} e^{-\frac{1}{2} A(x+\Delta)^{2}}\right) \partial_{\phi}\right), \\
& A_{3,5}^{2}=\operatorname{span}\left(\partial_{\tau}, e^{ \pm \frac{2 \tau}{B+1}} \partial_{x} \mp \frac{1}{B+1} e^{ \pm \frac{2 \tau}{B+1}} x \phi \partial_{\phi}, 2 e^{ \pm \frac{4 \tau}{B+1}} x \partial_{x} \pm e^{ \pm \frac{4 \tau}{B+1}}(B+1) \partial_{\tau} \mp\right. \\
& \left.\frac{2 e^{ \pm \frac{4 \tau}{B+1}}\left(x^{2} \mp 2\right) \phi}{1+B} \partial_{\phi}\right) \\
& A_{3,5}^{3}=\operatorname{span}\left(\partial_{\tau}, \partial_{x}-\left(\Gamma \phi+\frac{2 \Delta e^{-\Gamma x} x^{\frac{1-B}{1+B}}}{B+1}\right) \partial_{\phi},(x+2 \Gamma \tau) \partial_{x}+2 \tau \partial_{\tau}+\right. \\
& \left.\frac{1}{B+1}\left((2-\Gamma(B+1)(x+2 \Gamma \tau)) \phi-4 \Gamma \Delta \tau e^{-\Gamma x} x^{\frac{1-B}{B+1}}\right) \partial_{\phi}\right),
\end{aligned}
$$




$$
\begin{aligned}
& A_{3,5}^{4}=\operatorname{span}\left(\partial_{\tau}, \partial_{x}-\Gamma \phi \partial_{\phi},(x+2 \Gamma \tau) \partial_{x}+2 \tau \partial_{\tau}-\frac{((1+A) \Gamma(x+2 \Gamma \tau)-2) \phi}{A+1} \partial_{\phi}\right), \\
& A_{3,5}^{5}=\operatorname{span}\left(\partial_{\tau}, \partial_{x}-\left(B \phi+\frac{2 e^{-B x}}{x}\right) \partial_{\phi},(x+2 B \tau) \partial_{x}+2 \tau \partial_{\tau}-\right. \\
& \left.B\left(\frac{4 \tau e^{-B x}}{x}+(x+2 B \tau) \phi\right) \partial_{\phi}\right), \\
& A_{3,5}^{6}=\operatorname{span}\left(\partial_{\tau}, \partial_{x}+B \phi \partial_{\phi},(x-2 B \tau) \partial_{x}+2 \tau \partial_{\tau}+\left(B(x-2 B \tau) \phi-2 e^{B x}\right) \partial_{\phi}\right), \\
& A_{3,5}^{7}=\operatorname{span}\left(\partial_{\tau}, e^{ \pm 2 \tau} \partial_{x} \pm e^{ \pm 2 \tau}\left(e^{\mp \frac{1}{2} x^{2}}-\phi\right) x \partial_{\phi}, 2 e^{ \pm 4 \tau} x \partial_{x} \pm e^{ \pm 4 \tau} \partial_{\tau}+\right. \\
& \left.2 e^{ \pm 4 \tau}\left(\left( \pm 2 x^{2}+3\right) e^{\mp \frac{1}{2} x^{2}}-\left( \pm x^{2}+2\right) \phi\right) \partial_{\phi}\right) \\
& A_{3,5}^{8}=\operatorname{span}\left(\partial_{\tau}, e^{ \pm 2 \tau} \partial_{x}-\frac{e^{ \pm 2 t}\left( \pm u x(x+B)+2 e^{\mp \frac{x^{2}}{2}}\right)}{x+B} \partial_{\phi},\right. \\
& \left. \pm 2 e^{ \pm 4 \tau} x \partial_{x}+e^{ \pm 4 \tau} \partial_{t}+\frac{2 e^{ \pm 4 t}\left( \pm 2 B e^{\mp \frac{x^{2}}{2}}-u x^{2}(x+B)\right)}{x+B} \partial_{\phi}\right) \\
& A_{3,5}^{9}=\operatorname{span}\left(\partial_{\tau}, \partial_{x}-B \phi \partial_{\phi},(x+2 B \tau) \partial_{x}+2 \tau \partial_{\tau}-\left(\left(2+B x+2 B^{2} \tau\right) \phi-B^{2} e^{-B x}\right) \partial_{\phi}\right) \text {, } \\
& A_{3,5}^{10}=\operatorname{span}\left(\partial_{\tau}, e^{\mp 2 \tau} \partial_{x} \pm e^{\mp 2 \tau} x \phi \partial_{\phi}, 2 e^{\mp 4 \tau} x \partial_{x} \mp e^{\mp 4 \tau} \partial_{t}-2 e^{-4 \tau}\left(2 e^{ \pm \frac{1}{2} x^{2}} \mp x^{2} \phi\right) \partial_{\phi}\right) \text {, } \\
& A_{3,8}^{1}=\operatorname{span}\left(\partial_{\tau}, 2 \sqrt{B} x \cos (2 \sqrt{B} \tau) \partial_{x}+2 \sin (2 \sqrt{B} \tau) \partial_{\tau}+\right. \\
& \left(B\left(\Delta e^{-\frac{1}{2} \Gamma x^{2}} x^{A+\frac{3}{2}}+x^{2} \phi\right) \sin (2 \sqrt{B} \tau)+\right. \\
& \left.\sqrt{B}\left(2 \Gamma \Delta e^{-\frac{1}{2} \Gamma x^{2}} x^{A+\frac{3}{2}}+(2 A-1) \phi\right) \cos (2 \sqrt{B} t)\right) \partial_{\phi}, \\
& -2 \sqrt{B} x \sin (2 \sqrt{B} \tau) \partial_{x}+2 \cos (2 \sqrt{B} \tau) \partial_{t}+ \\
& \left(B\left(\Delta e^{-\frac{1}{2} \Gamma x^{2}} x^{A+\frac{3}{2}}+x^{2} \phi\right) \cos (2 \sqrt{B} \tau)-\right. \\
& \left.\left.\sqrt{B}\left(2 \Gamma \Delta e^{-\frac{1}{2} \Gamma x^{2}} x^{A+\frac{3}{2}}+(2 A-1) \phi\right) \sin (2 \sqrt{B} \tau)\right) \partial_{\phi}\right), \\
& A_{3,8}^{2}=\operatorname{span}\left(\partial_{\tau}, 2 \sqrt{|B|} e^{-2 \sqrt{|B|} \tau} x \partial_{x}-2 e^{-2 \sqrt{|B|} \tau} \partial_{\tau}+\right. \\
& e^{-2 \sqrt{|B|} \tau} \sqrt{|B|}\left((\sqrt{|B|}+2 \Gamma) \Delta e^{-\frac{1}{2} \Gamma x^{2}} x^{\frac{3}{2}+A}+\left(\sqrt{|B|} x^{2}+2 A-1\right) \phi\right) \partial_{\phi}, \\
& 2 \sqrt{|B|} e^{2 \sqrt{|B|} \tau} x \partial_{x}+2 e^{2 \sqrt{|B|} \tau} \partial_{\tau}- \\
& \left.e^{2 \sqrt{|B|} \tau} \sqrt{|B|}\left((\sqrt{|B|}-2 \Gamma) \Delta e^{-\frac{1}{2} \Gamma x^{2}} x^{\frac{3}{2}+A}+\left(\sqrt{|B|} x^{2}+1-2 A\right) \phi\right) \partial_{\phi}\right), \\
& A_{3,8}^{3}=\operatorname{span}\left(\partial_{\tau}, 2 x \partial_{x}+4 \tau \partial_{\tau}+\left(2 B \Gamma e^{-\frac{1}{2} B x^{2}} x^{\frac{3}{2}+A}+(2 A-1) \phi\right) \partial_{\phi},\right.
\end{aligned}
$$




$$
\begin{aligned}
& \left.4 x \tau \partial_{x}+4 \tau^{2} \partial_{\tau}-\left(\Gamma e^{-\frac{1}{2} B x^{2}}(1-4 B \tau) x^{\frac{3}{2}+A}+\left((2-4 A) \tau+x^{2}\right) \phi\right) \partial_{\phi}\right), \\
& A_{4}^{1}=\operatorname{span}\left(\partial_{\tau}, e^{A \tau} \phi \partial_{\phi}, 4 e^{\frac{1}{2}\left(A-\sqrt{A^{2}-16 B}\right) \tau} \partial_{x}+\right. \\
& \left(\sqrt{A^{2}-16 B}-A\right) e^{\frac{1}{2}\left(A-\sqrt{A^{2}-16 B}\right) \tau} x \phi \partial_{\phi}, 4 e^{\frac{1}{2}\left(\sqrt{A^{2}-16 B}+A\right) \tau} \partial_{x}- \\
& \left.\left(\sqrt{A^{2}-16 B}+A\right) e^{\frac{1}{2}\left(\sqrt{A^{2}-16 B}+A\right) \tau} x \phi \partial_{\phi}\right), \\
& A_{4}^{2}=\operatorname{span}\left(\partial_{\tau}, e^{A \tau} \phi \partial_{\phi}, 4 e^{\frac{A \tau}{2}} \sin \frac{\sqrt{\left|A^{2}-16 B\right|}}{2} \partial_{x}-\right. \\
& e^{\frac{A \tau}{2}} x\left(\sqrt{\left|A^{2}-16 B\right|} \cos \frac{\sqrt{\left|A^{2}-16 B\right|}}{2}+A \sin \frac{\sqrt{\left|A^{2}-16 B\right|}}{2}\right) \phi \partial_{\phi}, \\
& 4 e^{\frac{A \tau}{2}} \cos \frac{\sqrt{\left|A^{2}-16 B\right|} \tau}{2} \partial_{x}+ \\
& \left.e^{\frac{A \tau}{2}} x\left(\sqrt{\left|A^{2}-16 B\right|} \sin \frac{\sqrt{\left|A^{2}-16 B\right|} \tau}{2}-A \cos \frac{\sqrt{\left|A^{2}-16 B\right|} \tau}{2}\right) \phi \partial_{\phi}\right), \\
& A_{4}^{3}=\operatorname{span}\left(\partial_{\tau}, e^{ \pm \tau} \phi \partial_{\phi}, 4 e^{\frac{ \pm \tau}{2}} \partial_{x} \mp e^{\frac{ \pm \tau}{2}} x \phi \partial_{\phi}, 4 e^{\frac{ \pm \tau}{2}} \tau \partial_{x}-e^{\frac{\tau}{2}}(2 \pm \tau) x \phi \partial_{\phi}\right) \text {, } \\
& A_{4}^{4}=\operatorname{span}\left(\partial_{\tau}, e^{A \tau} \phi \partial_{\phi}, A \partial_{x}-B \phi \partial_{\phi}, 2 e^{A \tau} \partial_{x}-e^{A \tau}(A x-2 B \tau) \phi \partial_{\phi}\right) .
\end{aligned}
$$

Table 1: Group classification of Eq. (18)

\begin{tabular}{ll}
\hline Lie Algebra & $\hat{f}$ \\
\hline$A_{1}$ & $\forall \hat{f}$ \\
$A_{2,2}^{1}$ & $-\frac{e^{-\frac{1}{8} x^{2}}}{4 x^{A+2}}\left(x^{2}\left(\frac{1}{4} x^{2}+2 A-1\right) \psi+F(\psi)\right), \psi=e^{\frac{1}{8} x^{2}} x^{A} \phi, F^{\prime \prime} \neq 0$ \\
$A_{2,2}^{2}$ & $\frac{1}{x^{A+2}} F\left(x^{A} \phi\right), F^{\prime \prime} \neq 0$ \\
$A_{2,2}^{3}$ & $-e^{-\frac{1}{2}(A x+B) x}(A x(A x+B) \psi+F(\psi)), \psi=e^{\frac{1}{2}(A x+B) x} \phi, F^{\prime \prime} \neq 0$ \\
$A_{2,2}^{4}$ & $-(F(x)+A \log ((\Delta+x(\Gamma x+B)) \phi)) \phi, A, B^{2}+\Gamma^{2}+\Delta^{2} \neq 0$ \\
& $-e^{-\frac{1}{2} A(x+\Delta)^{2}} x^{-\frac{2 B}{B+1}}\left(\Gamma|\phi|^{-B}+\frac{A x^{2}\left(A(B+1)(x+\Delta)^{2}-B-5\right)}{B+1} \phi-\right.$ \\
$A_{3,5}^{1}$ & $\left.\quad \frac{E\left(A(B+1) x\left(x\left(A(B+1)(x+\Delta)^{2}-5-B\right)-4 E\right)+2(1-B)\right)}{(B+1)^{2}}\right)$ \\
& $\quad \psi=e^{\frac{1}{2} A(x+\Delta)^{2}} x^{-\frac{2}{B+1}} \phi+E, A, \Gamma \neq 0, B \neq 0,-1,-2$ \\
$A_{3,5}^{2}$ & $\frac{5+B \mp x^{2}}{(B+1)^{2}} \phi-A e^{\mp \frac{1}{2} x^{2}}|\phi|^{-B}, A \neq 0, B \neq 0,-1,-2$ \\
\hline
\end{tabular}


Table 1 - Continued from previous page

\begin{tabular}{|c|c|}
\hline Lie Algebra & $\hat{f}$ \\
\hline$A_{3,5}^{3}$ & $\begin{array}{c}-e^{-\Gamma x} x^{-\frac{2 B}{1+B}}\left(\frac{\Delta\left(2(B+1)-(\Gamma(B+1) x-2)^{2}\right)}{(B+1)^{2}}+A|\psi|^{-B}+\Gamma^{2} x^{2} \psi\right) \\
\psi=e^{A x} x^{-\frac{2}{1+B}} \phi+\Delta, A \neq 0, B \neq 0,-1,-2\end{array}$ \\
\hline$A_{3,5}^{4}$ & $-e^{-(A+1) B x}|\phi|^{-A}-B^{2} \phi, \quad A \neq 0,-1,-2$ \\
\hline$A_{3,5}^{5}$ & $-B^{2} u-\frac{A e^{-e^{B x} u-B x}}{x^{2}}-\frac{2 e^{-B x}(2 B x+1)}{x^{2}}, A \neq 0$ \\
\hline$A_{3,5}^{6}$ & $-e^{B x}\left(A e^{\psi}+B^{2} \psi\right), \psi=e^{-B x} \phi, A \neq 0$ \\
\hline$A_{3,5}^{7}$ & $\mp \frac{e^{\mp \frac{1}{2} x^{2}}}{4}\left(4 e^{ \pm x^{2}} u^{2}+\left(x^{2} \mp 11\right)\left(x^{2} \pm 1\right)\right)$ \\
\hline$A_{3,5}^{8}$ & $\mp e^{\mp \frac{1}{2} x^{2}}\left(\frac{A}{(x+B)^{2}} e^{-\psi} \pm\left( \pm x^{2}-1\right) \psi+\frac{2(1 \mp 2 B(x+B))}{(x+B)^{2}}\right), \psi=e^{ \pm \frac{1}{2} x^{2}} \phi, A \neq 0$ \\
\hline$A_{3,5}^{9}$ & $-e^{B x} \phi^{2}-\frac{B^{4}}{4} e^{-B x}$ \\
\hline$A_{3,5}^{10}$ & $\mp e^{ \pm \frac{1}{2} x^{2}}\left(A e^{\psi}+\left( \pm x^{2}+1\right) \psi-4\right), \psi=e^{\mp \frac{1}{2} x^{2}} \phi, A \neq 0$ \\
\hline$A_{3,8}^{1}$ & $\begin{array}{l}-e^{-\frac{1}{2} \Gamma x^{2}} x^{A-\frac{5}{2}} \psi\left(4 A \log |\psi|-\frac{1}{4} x^{2}\left(B x^{2}+8 A \Gamma\right)\right)+ \\
\frac{1}{4} \Delta e^{-\frac{1}{2} \Gamma x^{2}} x^{A-\frac{5}{2}}\left(3-8 A+4\left(\Gamma x^{2}-A\right)^{2}\right), \psi=e^{\frac{1}{2} \Gamma x^{2}} x^{\frac{1}{4}(2-4 A)} \phi+\Delta \\
\quad A \neq 0, B>0\end{array}$ \\
\hline$A_{3,8}^{2}$ & $\begin{array}{l}-e^{-\frac{1}{2} \Gamma x^{2}} x^{A-\frac{5}{2}} \psi\left(4 A \log |\psi|-\frac{1}{4} x^{2}\left(B x^{2}+8 A \Gamma\right)\right)+ \\
\frac{1}{4} \Delta e^{-\frac{1}{2} \Gamma x^{2}} x^{A-\frac{5}{2}}\left(3-8 A+4\left(\Gamma x^{2}-A\right)^{2}\right), \psi=e^{\frac{1}{2} \Gamma x^{2}} x^{\frac{1}{4}(2-4 A)} \phi+\Delta \\
\quad A \neq 0, B<0\end{array}$ \\
\hline$A_{3,8}^{3}$ & $\begin{array}{c}2 e^{-\frac{1}{2} B x^{2}} x^{A-\frac{5}{2}} \psi\left(A B x^{2}-2 A \log |\psi|\right)+\frac{1}{4} \Gamma e^{-\frac{1}{2} B x^{2}} x^{A-\frac{5}{2}}(3-8 A)+ \\
\Gamma e^{-\frac{1}{2} B x^{2}} x^{A-\frac{5}{2}}\left(B x^{2}-A\right)^{2}, \psi=e^{\frac{1}{2} B x^{2}} x^{\frac{1}{4}(2-4 A)} \phi+\Gamma, A \neq 0\end{array}$ \\
\hline$A_{4}^{1}$ & $\phi\left(A \log |\phi|+B x^{2}\right), A, B \neq 0, A^{2}-16 B>0$ \\
\hline$A_{4}^{2}$ & $\phi\left(A \log |\phi|+B x^{2}\right), A, B \neq 0, A^{2}-16 B<0$ \\
\hline$A_{4}^{3}$ & $\phi\left(\frac{1}{16} x^{2} \pm \log |\phi|\right)$ \\
\hline$A_{4}^{4}$ & $\phi(A \log |\phi|+B x), \quad A \neq 0$ \\
\hline
\end{tabular}

In Table 1 we have denoted by $A, B, \Gamma, \Delta, E, \ldots$ arbitrary real constants, some of them satisfying the stated relations and $\mathcal{F}_{1}(x)$ is an arbitrary real function.

Remark 3.3. By investigating the algebraic structure of the symmetry sets 
we found that, the algebras $A_{3,5}^{1}$ and $A_{3,5}^{2}$, the algebras $A_{3,5}^{3}$ and $A_{3,5}^{4}$ and the algebras $A_{3,5}^{5}$ and $A_{3,5}^{6}$ are connected via an additional equivalence transformation.

\section{Examples of invariant solutions}

Having obtained the complete group classification for Eq. (18), and consequently for Eq. (5), we can look for invariant solutions under the terminal condition (8) and the barrier option condition (9): Given a specific Lie algebra from Table 1, the appropriate subalgebra, and the functions $H(t), R(t)$ for the barrier option problem, admitted by each problem are determined using the two required conditions (13), (14) and (15), (16) adapted now to Eq. (18). Namely,

$$
\begin{gathered}
\left.\mathfrak{X}\left(\tau-T^{\prime}\right)\right|_{\tau=T^{\prime}} \equiv 0, \\
\left.\mathfrak{X}\left(\phi-e^{-\frac{1}{b^{2}}(a x+1)}\right)\right|_{\tau=T^{\prime}, \phi=e^{-\frac{1}{b^{2}}(a x+1)}} \equiv 0,
\end{gathered}
$$

where $T^{\prime}=-\frac{b^{2}}{2} T$, and

$$
\begin{gathered}
\left.\mathfrak{X}(x-H(\tau))\right|_{x=H(\tau)} \equiv 0, \\
\left.\mathfrak{X}\left(\phi-e^{-\frac{1}{b^{2}}(a H(\tau)+R(\tau))}\right)\right|_{x=H(\tau), \phi=e^{-\frac{a}{b^{2}} H(\tau)-\frac{1}{b^{2}} R(\tau)}} \equiv 0 .
\end{gathered}
$$

Finally, by using that subalgebra similarity solutions are constructed as per usual.

\subsection{The terminal condition}

Due to the restrictions imposed by the terminal condition all the Lie algebras of dimension less than 4 either they do not yield a suitable subalgebra or the found subalgebra gives trivial similarity solutions (constants or functions depending only on $t$ ). Hence, we continue by presenting a nontrivial similarity solution for the Lie algebra $A_{4}^{1}$.

Let the arbitrary element of the Lie algebra be $\mathfrak{X}=\mathbf{c}_{1} \mathfrak{X}_{1}+\mathbf{c}_{2} \mathfrak{X}_{2}+\mathbf{c}_{3} \mathfrak{X}_{3}+$ $\mathbf{c}_{4} \mathfrak{X}_{4}$, where $\mathfrak{X}_{i}$ are the basis elements of the chosen Lie algebra. Using (19) and (20) we obtain the constraints:

$$
\mathbf{c}_{1}=0
$$


and

$$
\begin{gathered}
\frac{1}{4 b^{2}} e^{-\frac{4+4 a x-2 b^{2} T^{\prime}\left(A+\sqrt{A^{2}-16 B}\right)}{4 b^{2}}}\left(b^{2} \sqrt{A^{2}-16 B} x\left(\mathbf{c}_{2}-e^{-T^{\prime} \sqrt{A^{2}-16 B}} \mathbf{c}_{3}\right)-\right. \\
\left.\left(4 a-b^{2} A x\right)\left(\mathbf{c}_{2}+e^{-T^{\prime} \sqrt{A^{2}-16 B}} \mathbf{c}_{3}\right)-4 b^{2} e^{-\frac{1}{2} T^{\prime}\left(\sqrt{A^{2}-16 B}-A\right)} \mathbf{c}_{4}\right)=0 .
\end{gathered}
$$

From the above two constraints we have the subalgebra defined by

$$
\begin{aligned}
& \mathbf{c}_{1}=0, \\
& \mathbf{c}_{3}=\frac{e^{T^{\prime} \sqrt{A^{2}-16 B}}\left(A+\sqrt{A^{2}-16 B}\right) \mathbf{c}_{2}}{\sqrt{A^{2}-16 B}-A}, \\
& \mathbf{c}_{4}=\frac{2 a e^{\frac{1}{2} T^{\prime}}\left(\sqrt{A^{2}-16 B}-A\right) \sqrt{A^{2}-16 B} \mathbf{c}_{2}}{b^{2}\left(A-\sqrt{A^{2}-16 B}\right)} .
\end{aligned}
$$

It follows that the invariant surface condition is

$$
\begin{aligned}
& 2\left(a e^{\frac{1}{2}\left(T^{\prime}-\tau\right)\left(\sqrt{A^{2}-16 B}-A\right)} \sqrt{A^{2}-16 B}+\right. \\
& \left.2 b^{2}\left(e^{\left(T^{\prime}-\tau\right) \sqrt{A^{2}-16 B}}-1\right) B x\right) \phi+b^{2}\left(\left(e^{\left(T^{\prime}-\tau\right) \sqrt{A^{2}-16 B}}-1\right) A+\right. \\
& \left.\left(1+e^{\left(T^{\prime}-\tau\right) \sqrt{A^{2}-16 B}}\right) \sqrt{A^{2}-16 B}\right) \phi_{x}=0 .
\end{aligned}
$$

Eq. (24) has the solution

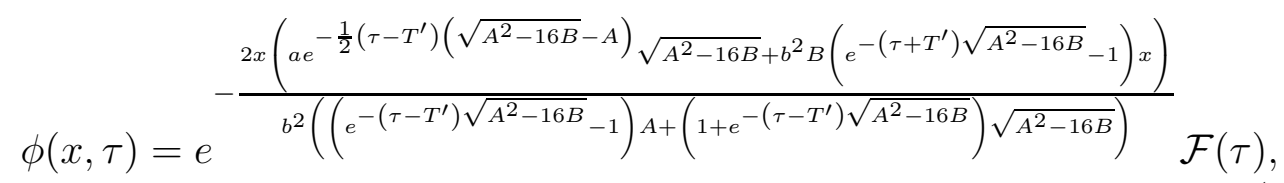

where $\mathcal{F}(\tau)$ is an arbritrary real function. Using the above similarity solution Eq. (18) is reduced to the ODE

$$
\begin{gathered}
\left(2 a^{2} e^{\left(\tau-T^{\prime}\right)\left(A-\sqrt{A^{2}-16 B}\right)}\left(A^{2}-16 B\right)+b^{4}\left(2 B\left(\sqrt{A^{2}-16 B}-A\right)-\right.\right. \\
2 e^{-2\left(\tau-T^{\prime}\right) \sqrt{A^{2}-16 B}} \sqrt{A^{2}-16 B} B+A \Delta\left(A-\sqrt{A^{2}-16 B}\right)+ \\
e^{-2\left(\tau-T^{\prime}\right) \sqrt{A^{2}-16 B}} A \sqrt{A^{2}-16 B} \Delta-8 B \Delta+e^{-2\left(\tau-T^{\prime}\right) \sqrt{A^{2}-16 B}}\left(A^{2} \Delta\right. \\
\left.-2 A B-8 B \Delta)+4 e^{-\left(\tau-T^{\prime}\right) \sqrt{A^{2}-16 B}}(A B-4 B \Delta)\right)+
\end{gathered}
$$




$$
\begin{gathered}
b^{4} A\left(\left(1+e^{-2\left(\tau-T^{\prime}\right) \sqrt{A^{2}-16 B}}\right) A^{2}+\left(e^{-2\left(\tau-T^{\prime}\right) \sqrt{A^{2}-16 B}}-1\right) A \sqrt{A^{2}-16 B}-\right. \\
\left.\left.8\left(1+e^{-\left(\tau-T^{\prime}\right) \sqrt{A^{2}-16 B}}\right)^{2} B\right) \log |\mathcal{F}|\right) \mathcal{F}+ \\
b^{4}\left(\left(1+e^{-2\left(\tau-T^{\prime}\right) \sqrt{A^{2}-16 B}}\right) A^{2}+\left(e^{-2\left(\tau-T^{\prime}\right) \sqrt{A^{2}-16 B}}-1\right) A \sqrt{A^{2}-16 B}-\right. \\
\left.8\left(1+e^{-\left(\tau-T^{\prime}\right) \sqrt{A^{2}-16 B}}\right)^{2} B\right) \mathcal{F}^{\prime}=0 .
\end{gathered}
$$

Solving it, by also setting $A=3$ and $B=1 / 2$, we obtain the solution

$$
\mathcal{F}(\tau)=e^{\frac{e^{3 \tau}\left(8 b^{4} e^{-4 \tau}+12 a^{2} e^{-4 T^{\prime}}+3 b^{4} e^{-\tau-3 T^{\prime}}-3 e^{-2 T^{\prime}}\left(b^{4} e^{-2 \tau}-24 c\right)-2 e^{-\tau-T^{\prime}}\left(5 b^{4} e^{-2 \tau}+72 c\right)\right)}{24 b^{4}\left(2 e^{-\tau}-e^{-T^{\prime}}\right)}} \times
$$

where $c$ is the constant of integration. In order the similarity solution found, consisting of Eqs. (25) and (26), to satisfy the terminal condition the constant of integration $c$ must be

$$
c=\frac{1}{288} e^{-2 T^{\prime}}\left(48 a^{2}+96 b^{2}-8 b^{4}-6 b^{4} T^{\prime}\right) .
$$

Overall, after returning back to the initial Eq. (5), we have that the function

$$
\begin{gathered}
u(x, t)=\frac{1}{96 b^{2}\left(2 e^{\frac{b^{2} t}{2}}-e^{\frac{b^{2} T}{2}}\right)} e^{-\frac{3 b^{2} t}{2}}\left(3 b^{6}\left(e^{2 b^{2} T}-2 e^{\frac{1}{2} b^{2}(t+3 T)}\right) T-\right. \\
96 a^{2}\left(e^{2 b^{2} T}-e^{\frac{1}{2} b^{2}(t+3 T)}\right)-96 b^{2}\left(e^{2 b^{2} T}-2 e^{\frac{1}{2} b^{2}(t+3 T)}+2 a e^{2 b^{2} t} x-\right. \\
\left.a e^{b^{2}(t+T)} x-a e^{\frac{1}{2} b^{2}(3 t+T)} x\right)+4 b^{4}\left(2 e^{2 b^{2} T}+3 e^{b^{2}(t+T)}-7 e^{\frac{1}{2} b^{2}(t+3 T)}+\right. \\
\left.4 e^{2 b^{2} t}\left(3 x^{2}-2\right)-2 e^{\frac{1}{2} b^{2}(3 t+T)}\left(6 x^{2}-5\right)\right)- \\
\left.6 b^{4}\left(e^{2 b^{2} T}-2 e^{\frac{1}{2} b^{2}(t+3 T)}\right) \log \left|2 e^{\frac{b^{2} t}{2}}-e^{\frac{b^{2} T}{2}}\right|\right),
\end{gathered}
$$

is a similarity solution of the PDE

$$
u_{t}(x, t)=a u_{x}+\frac{1}{2} u_{x}^{2}-\frac{1}{2} b^{2} u_{x x}+\frac{a^{2}}{2}+\frac{1}{2} b^{4}\left(\frac{x^{2}}{2}-\frac{3(a x+u)}{b^{2}}\right) .
$$




\subsection{The barrier option condition}

In what follows we present, in detail, a similarity solution using the Lie algebra $A_{4}^{1}$ for a specific choice of the respective function $\hat{f}$. Then, we briefly give examples of similarity solutions for Lie algebras of lower dimension.

Again, let the arbitrary element of the Lie algebra be $\mathfrak{X}=\mathbf{c}_{1} \mathfrak{X}_{1}+\mathbf{c}_{2} \mathfrak{X}_{2}+$ $\mathbf{c}_{3} \mathfrak{X}_{3}+\mathbf{c}_{4} \mathfrak{X}_{4}$. Using (21) and (22) we obtain the ODE for $H(\tau)$

$$
4 e^{\frac{1}{2}\left(A-\sqrt{A^{2}-16 B}\right) \tau}\left(\mathbf{c}_{3}+e^{\sqrt{A^{2}-16 B} \tau} \mathbf{c}_{4}\right)-\mathbf{c}_{1} H^{\prime}=0,
$$

with solution

$$
\begin{aligned}
H(\tau)= & \frac{e^{\frac{1}{2}\left(A-\sqrt{A^{2}-16 B}\right) \tau}}{2 B \mathbf{c}_{1}}\left(\sqrt{A^{2}-16 B}\left(\mathbf{c}_{3}-e^{\sqrt{A^{2}-16 B} \tau} \mathbf{c}_{4}\right)+\right. \\
& \left.A\left(\mathbf{c}_{3}+e^{\sqrt{A^{2}-16 B} \tau} \mathbf{c}_{4}\right)+2 e^{-\frac{1}{2}\left(A-\sqrt{A^{2}-16 B}\right) \tau} B \mathbf{c}_{1} \mathbf{c}_{5}\right), \mathbf{c}_{1} \neq 0,
\end{aligned}
$$

where $\mathbf{c}_{5}$ is the constant of integration, and an ODE for $R(\tau)$

$$
\begin{gathered}
4 a B \mathbf{c}_{1} e^{\frac{1}{2}\left(A-\sqrt{A^{2}-16 B}\right) \tau}\left(\mathbf{c}_{3}+e^{\sqrt{A^{2}-16 B} \tau} \mathbf{c}_{4}\right)+ \\
b^{2}\left(B \left(\mathbf{c}_{1} \mathbf{c}_{3} \mathbf{c}_{5}\left(\sqrt{A^{2}-16 B}-A\right) e^{\frac{1}{2}\left(A-\sqrt{A^{2}-16 B}\right) \tau}-\right.\right. \\
\mathbf{c}_{1} \mathbf{c}_{4} \mathbf{c}_{5}\left(\sqrt{A^{2}-16 B}+A\right) e^{\frac{1}{2}\left(\sqrt{A^{2}-16 B}+A\right) \tau}-8 \mathbf{c}_{3}{ }^{2} e^{\left(A-\sqrt{A^{2}-16 B}\right) \tau}- \\
\left.\left.8 \mathbf{c}_{4}{ }^{2} e^{\left(\sqrt{A^{2}-16 B}+A\right) \tau}+\left(\mathbf{c}_{1} \mathbf{c}_{2}+16 \mathbf{c}_{3} \mathbf{c}_{4}\right) e^{A \tau}\right)-2 A^{2} \mathbf{c}_{3} \mathbf{c}_{4} e^{A \tau}\right)+B \mathbf{c}_{1}^{2} R^{\prime}(t)=0,
\end{gathered}
$$

with solution

$$
\begin{gathered}
R(\tau)=\frac{1}{B \mathbf{c}_{1}^{2}}\left(\frac { 1 } { 2 } e ^ { \frac { 1 } { 2 } ( A - \sqrt { A ^ { 2 } - 1 6 B } ) \tau } \left(b ^ { 2 } \left(4 B \mathbf{c}_{1}\left(\mathbf{c}_{4} \mathbf{c}_{5} e^{\sqrt{A^{2}-16 B} \tau}+\mathbf{c}_{3}\right)+\right.\right.\right. \\
e^{\frac{1}{2}\left(A-\sqrt{A^{2}-16 B}\right) \tau}\left(A\left(\mathbf{c}_{4}{ }^{2} e^{2 \sqrt{A^{2}-16 B} \tau}+\mathbf{c}_{3}{ }^{2}\right)+\right. \\
\left.\left.\sqrt{A^{2}-16 B}\left(\mathbf{c}_{3}{ }^{2}-\mathbf{c}_{4}{ }^{2} e^{2 \sqrt{A^{2}-16 B} \tau}\right)\right)\right)-a \mathbf{c}_{1}\left(A\left(\mathbf{c}_{4} e^{\sqrt{A^{2}-16 B} \tau}+\mathbf{c}_{3}\right)+\right. \\
\left.\left.\sqrt{A^{2}-16 B}\left(\mathbf{c}_{3}-\mathbf{c}_{4} e^{\sqrt{A^{2}-16 B} \tau}\right)\right)\right)-\frac{b^{2} B\left(\mathbf{c}_{1} \mathbf{c}_{2}+16 \mathbf{c}_{3} \mathbf{c}_{4}\right) e^{A \tau}}{A}+ \\
\left.2 A b^{2} \mathbf{c}_{3} \mathbf{c}_{4} e^{A \tau}\right)+\mathbf{c}_{6},
\end{gathered}
$$

where $\mathbf{c}_{6}$ is the constant of integration. 
The solution $H$ found above includes the barrier function usually used in literature, (10). Indeed, by setting

$$
\mathbf{c}_{1}=-\frac{2 b^{2} e^{\alpha T} \mathbf{c}_{3}}{\alpha \beta K}, \mathbf{c}_{4}=\mathbf{c}_{5}=0
$$

and

$$
B=-\frac{b^{2} \alpha A+2 \alpha^{2}}{2 b^{4}},
$$

the functions $H$ and $R$ became respectively

$$
H(\tau)=K \beta e^{-\frac{2 \alpha\left(\tau+b^{2} T / 2\right)}{b^{2}}}
$$

and

$$
R(\tau)=\frac{1}{2} \beta K\left(\frac{\alpha \mathbf{c}_{2} e^{A \tau-\alpha T}}{A \mathbf{c}_{3}}-e^{-2 \alpha\left(\frac{2 \tau}{b^{2}}+T\right)}\left(2 a e^{\alpha\left(\frac{2 \tau}{b^{2}}+T\right)}+\alpha \beta K\right)\right)+\mathbf{c}_{6},
$$

where we have assumed that $A b^{2}>-4 \alpha$. Furthermore, by setting

$$
\mathbf{c}_{2}=\mathbf{c}_{6}=0
$$

$R$ simplifies to

$$
R(\tau)=-\frac{1}{2} \beta K e^{-2 \alpha\left(\frac{2 \tau}{b^{2}}+T\right)}\left(2 a e^{\alpha\left(\frac{2 \tau}{b^{2}}+T\right)}+\alpha \beta K\right) .
$$

It follows that the invariant surface condition is now

$$
b^{4} e^{\frac{\alpha\left(2 \tau+b^{2} T\right)}{b^{2}}} \phi_{\tau}+2 \alpha \beta K\left(\alpha x \phi-b^{2} \phi_{x}\right)=0 .
$$

Eq. (29) has the solution

$$
\phi(x, \tau)=e^{\frac{\alpha x^{2}}{2 b^{2}}} \mathcal{F}\left(\frac{b^{2} e^{-\frac{2 \alpha \tau}{b^{2}}-\alpha T}\left(e^{\frac{2 \alpha \tau}{b^{2}}+\alpha T} x-K \beta\right)}{2 \alpha \beta K}\right) .
$$

Using the above similarity solution Eq. (18) is reduced to the ODE

$$
4 \alpha^{2} \beta^{2} K^{2}\left(2 \alpha \zeta \mathcal{F}^{\prime}+\mathcal{F}\left(\alpha+A b^{2} \log |\mathcal{F}|+b^{2} \Delta\right)\right)+b^{6} \log |\mathcal{F}|^{\prime \prime}=0,
$$


where $\zeta=\frac{b^{2} e^{-\frac{2 \alpha t}{b^{2}}-\alpha T}\left(x e^{\frac{2 \alpha t}{b^{2}}+\alpha T}-\beta K\right)}{2 \alpha \beta K}$. A special solution of the above ODE is the following

$$
\mathcal{F}(\zeta)=\exp \left(\frac{2 \alpha+A b^{2}-2 b^{2} \Delta}{2 A b^{2}}-\frac{\alpha^{2} \beta^{2} K^{2}\left(4 \alpha+A b^{2}\right)}{b^{6}} \zeta^{2}\right) .
$$

In addition, for the above solution to satisfy the condition $\phi=e^{-\frac{a H(\tau)+R(\tau)}{b^{2}}}$ the constant $\Delta$ must be set equal to $\frac{A}{2}+\frac{\alpha}{b^{2}}$.

Finally, after returning back to the initial Eq. (5), we have that the function

$$
u(x, t)=\frac{1}{4}\left(\left(4 \alpha+A b^{2}\right)\left(x-\beta K e^{\alpha(t-T)}\right)^{2}-2 \alpha x^{2}\right)-a x,
$$

is a similarity solution of the PDE

$$
\begin{aligned}
& u_{t}(x, t)=a u_{x}+\frac{1}{2} u_{x}^{2}-\frac{1}{2} b^{2} u_{x x}+\frac{1}{2} a^{2}- \\
& \frac{1}{2} A b^{2}(a x+u)+\frac{1}{4}\left(2 \alpha+A b^{2}\right)\left(b^{2}-\alpha x^{2}\right) .
\end{aligned}
$$

By using now the Lie algebra $A_{2,2}^{1}$ we can obtain the similarity solution

$$
u(x, t)=b^{2}\left(-\log \left(e^{-\frac{x^{2}}{8}} \sqrt{x} \log \left(8 \sec ^{2}\left(\frac{1}{4}\left(b^{2} t-2 c_{3}\right)+\log x\right)\right)\right)\right)-a x
$$

for the PDE

$$
\begin{aligned}
u_{t}(x, t)=a u_{x} & +\frac{1}{2} u_{x}^{2}-\frac{1}{2} b^{2} u_{x x}+ \\
& \frac{1}{32}\left(16 a^{2}+b^{4}\left(8-\frac{4 \sqrt{x} e^{\frac{e^{\frac{x^{2}}{8}-\frac{a x+u}{b^{2}}}}{\sqrt{x}}+\frac{a x+u}{b^{2}}-\frac{x^{2}}{8}}+x^{4}-4}{x^{2}}\right)\right) .
\end{aligned}
$$

And finally by using the Lie algebra $A_{3,5}^{9}$ we can obtain the similarity solution

$$
u(x, t)=b^{2}\left(-\log \left(\frac{3}{2} e^{-3 x}\left(\frac{4}{\left(3 b^{2} t-6 c_{1}+x\right)^{2}}+3\right)\right)\right)-a x
$$


for the PDE

$$
\begin{aligned}
& u_{t}(x, t)=a u_{x}+\frac{1}{2} u_{x}^{2}-\frac{1}{2} b^{2} u_{x x}+ \\
& \frac{1}{8}\left(4 a^{2}-b^{4}\left(77 \sinh \left(\frac{a x-3 b^{2} x+u}{b^{2}}\right)+85 \cosh \left(\frac{a x-3 b^{2} x+u}{b^{2}}\right)\right)\right) .
\end{aligned}
$$

\section{Conclusion}

In the present paper a generalization of the Heath equation (5) was proposed and studied under the view of the modern group analysis. To that end, we harnessed the advantage of being able to connect it with the heat equation with nonlinear course, a well known and studied equation. This fact substantially simplifies the task of classifying it and obtaining its point symmetries.

Through this classification interesting cases, from the point of view of symmetries, arise. Nonlinear equations in general have few or no symmetries so cases that augment the set of symmetries at disposal are like an oasis in the desert. After all, it is evident in the related literature that a dynamical system possessing an ample number of symmetries is more probable to relate with a physical system or model a more realistic process. Furthermore, in the case that we wish to study a boundary problem, because of the fact that not all of the symmetries admit the boundary conditions, some of the symmetries will be excluded. Hence the bigger the set of symmetries the bigger the probability that some will survive the scrutiny of the boundary conditions and give an invariant solution for the problem in its entirety.

This is evident for the terminal condition where due to the restrictions imposed by it only four dimensional algebras are able to yield nontrivial solutions.

Things are different when the barrier option is considered, because of the two functions $H(t), R(t)$ a broader range of cases can yield interesting solutions. It is worth mentioning at this point that, as can be seen by the second example, the barrier function $H$ usually used in the related literature is admitted by the symmetries. This fact reinforces further our belief that symmetries methods can be a valuable tool in investigating this kind of financial problems.

Indeed, the insight provided through the above symmetry analysis might prove practical to anyone looking for a more realistic economic model without 
departing from the reasoning behind the Heath model. Moreover, when one studies more exotic kinds of options, e.g. options that have gained ground in the Asian markets which in turn play an ever increasing role in the world market. The nonlinear variants of the traditional model given here might turn the table in that respect. We leave to the interested reader the possible economical interpretation and use of the obtained results.

\section{Acknowledgements}

We would like to thank the reviewers for their useful remarks that helped the manuscript to reach its present form. We would also like to thank FAPESP for the PostDoc grant (Proc. \#2011/05855-9) giving S. Dimas the opportunity to visit IMECC-UNICAMP where this work was carried out.

\section{References}

[1] F. Black, M. Scholes, The pricing of options and corporate liabilities, J. Polit. Econ. 81 (1973) 637-659.

[2] F. A. Longstaff, A nonlinear general equilibrium model of the term structure of interest rates, J. Financ. Econ. 23 (1989) 195-224.

[3] O. Vasicek, An equilibrium characterization of the term structure, J. Financ. Econ. 5 (1977) 177-188.

[4] J. C. Cox, J. E. Ingersoll, S. A. Ross, An intertemporal general equilibrium model of asset prices, Econometrica 53 (1985) 363-384.

[5] D. Heath, E. Platin, M. Schweizer, Numerical comparison of local riskminimisation and mean-variance hedging, in: E. Jouini, C. Jajusa, M. Murek (Eds.), Option Pricing, Interest Rates and Risk Management, CUP, Cambridge, 2001, pp. 509-537.

[6] R. K. Gazizov, N. H. Ibragimov, Lie symmetry analysis of differential equations in finance, Nonlinear Dynam. 17 (1998) 387-407.

[7] S. Dimas, K. Andriopoulos, D. Tsoubelis, P. G. L. Leach, Complete specification of some partial differential equations that arise in financial mathematics, J. Nonlinear Math. Phys. 16, s-1 (2009) 73-92. 
[8] O. Sinkala, P. Leach, J. O'Hara, Invariance properties of a general bond-pricing equation, J. Diff. Eq. 244 (2008) 2820-2835.

[9] Y. Bozhkov, S. Dimas, Group analysis of a semi-linear general bondpricing equation, 2014. Submitted.

[10] V. Naicker, K. Andriopoulos, P. G. L. Leach, Symmetry Reductions of a Hamilton-Jacobi-Bellman Equation Arising in Financial Mathematics, J. Nonlinear Math. Phys. 12 (2005) 268-283.

[11] G. W. Bluman, S. Kumei, Symmetries and differential equations, Springer, New York, 1989.

[12] L. Ovsiannikov, Group Analysis of Differential Equations, Academic Press, $1^{\text {st }}$ edition, 1982. 432 pages.

[13] N. H. Ibragimov, Equivalence groups and invariants of linear and nonlinear equations, Archives of ALGA 4 (2009) 41-100.

[14] R. O. Popovych, H. Eshraghi, Admissible point transformations of nonlinear Schrödinger equations, in: N. Ibragimov, C. Sophocleous, P. Damianou (Eds.), Proceedings of the $10^{\text {th }}$ International Conference in MOdern GRoup ANalysis, 2005, pp. 167-174.

[15] V. Romano, M. Torrisi, Application of weak equivalence transformations to a group analysis of a drift-diffusion model, J. Phys. A: Math. Gen. 32 (1999) 7953.

[16] Y. Bozhkov, S. Dimas, Group classification of a generalized BlackScholes-Merton equation, Commun. Nonlinear Sci. Numer. Simul. 19 (2014) 2200-2211.

[17] R. Cherniha, M. Serov, I. Rassokha, Lie symmetries and form-preserving transformations of reaction-diffusion-convection equations, J. Math. Anal. Appl. 342 (2008) 1363-1379.

[18] E. D. S. Cardoso-Bilho, A. Bilho, R. O. Popovych, Enhanced preliminary group classification of a class of generalized diffusion equations, Commun. Nonlinear Sci. Numer. Simul. 16 (2011) 3622-3638. 
[19] N. M. Ivanova, R. O. Popovych, C. Sophocleous, Group analysis of variable coefficient diffusion-convection equations. I. Enhanced group classification, Lobachevskii J. Math. 31 (2010) 100-122.

[20] O. O. Vaneeva, R. O. Popovych, C. Sophocleous, Enhanced group analysis and exact solutions of variable coefficient semilinear diffusion equations with a power source, Acta Appl. Math. 106 (2009) 1-46.

[21] F. Black, M. Scholes, The valuation of option contracts and a test of market efficiency, J. Finance 27 (1972) 399-417.

[22] R. C. Merton, On the pricing of corporate debt: The risk structure of interest rates, J. Finance 29 (1974) 449-470.

[23] O. Ugur, Introduction to computational finance, Imperial College Press and World Scientific, 2008.

[24] J. O'Hara, Lecture notes on exotic options, 2011. Http://courses.essex.ac.uk/cf/cf966/.

[25] Y. K. Kwok, Mathematical Models of Financial Derivatives, Springer, 2nd edition, 2008.

[26] J. O'Hara, C. Sophocleous, P. G. L. Leach, Symmetry analysis of a model for the exercise of a barrier option, Commun. Nonlinear Sci. Numer. Simul. 18 (2013) 2367-2373.

[27] P. J. Olver, Applications of Lie Groups to Differential Equations, volume 107 of Graduate Texts in Mathematics, Springer, New York, $2^{\text {nd }}$ edition, 2000.

[28] A. K. Head, Lie, a pc program for Lie analysis of differential equations, Comput. Phys. Comm. 77 (1993) 241-248.

[29] M. Nucci, Interactive REDUCE programs for calculating Lie point, non-classical, Lie-Bäcklund, and approximate symmetries of differential equations: manual and floppy disk, in: N. Ibragimov (Ed.), CRC Handbook of Lie Group Analysis of Differential Equations. Vol. III:New Trends, CRC Press, 1996, pp. 415-481. 
[30] M. Nucci, Interactive REDUCE programs for calculating classical, nonclassical and Lie-Bäcklund symmetries for differential equations, in: W. Ames, P. Van der Houwen (Eds.), Computational and Applied Mathematics II. Differential Equations, Elsevier, 1992, pp. 345-350.

[31] G. Baumann, Symmetry Analysis of Differential Equations with Mathematica, Telos/Springer, New York, 2000.

[32] W. Reasearch, Inc., Mathematica Edition: Version 8.0, Wolfram Reasearch, Inc., Champaign, Illinois, 2010.

[33] S. Dimas, Partial differential equations, algebraic computing and nonlinear systems, Ph.D. Thesis, University of Patras, Patras, Greece, 2008.

[34] S. Dimas, D. Tsoubelis, SYM: A new symmetry-finding package for Mathematica, in: N. Ibragimov, C. Sophocleous, P. Damianou (Eds.), The $10^{\text {th }}$ International Conference in MOdern GRoup ANalysis, University of Cyprus, Nicosia, 2005, pp. 64-70.

[35] S. Dimas, D. Tsoubelis, A new Mathematica-based program for solving overdetermined systems of PDEs, in: Y. Papegay (Ed.), Applied Mathematica, Electronic Proceedings of the Eighth International Mathematica Symposium (IMS'06), France: INRIA, Avignon, France, 2006. ISBN 2-7261-1289-7.

[36] N. H. Ibragimov, Transformation Groups Applied to Mathematical Physics, Mathematics and its Applications, Springer, $1^{\text {st }}$ edition, 1985.

[37] P. E. Hydon, Symmetry Methods for Differential Equations, Cambridge Texts in Applied Mathematics, Cambridge University Press, Cambridge, $1^{\text {st }}$ edition, 2000.

[38] H. Stephani, Differential Equations: Their Solution Using Symmetries, Cambridge University Press, Cambridge, $1^{\text {st }}$ edition, 1990. Editor: MacCallum, Malcolm.

[39] R. Z. Zhdanov, V. I. Lahno, Group classification of heat conductivity equations with a nonlinear source., J. Phys. A: Math. Gen. 32 (1999) 7405-7418. 